\title{
Respuesta educativa individualizada a las dificultades en el proceso de enseñanza-aprendizaje de las ciencias en la etapa de secundaria
}

\author{
Marta Vidal, Esther Cascarosa y Víctor Roda. Universidad de Zaragoza \\ Recepción: 14 de octubre de 2016 | Revisión: 16 de octubre de 2016 | Aceptado: 26 octubre de 2016 \\ Correspondencia: Esther Cascarosa | Email: ecascano@unizar.es \\ Citar: Vidal, M., Cascarosa, E. y Roda, V. (2016). Respuesta educativa individualizada a las dificultades en el proceso de enseñanza- \\ aprendizaje de las ciencias en la etapa de secundaria. ReiDoCrea, 5, 263-277.
}

\begin{abstract}
Resumen: Se presenta un estudio de casos cuyo objetivo ha sido proponer nuevas metodologías de trabajo adaptadas a las necesidades de los alumnos. Previo al diseño de estas, se ha llevado a cabo una revisión bibliográfica en profundidad sobre las pautas de actuación del profesorado ante las dificultades de aprendizaje de las ciencias en alumnos adolescentes. Como consecuencia, ha surgido la necesidad de acompañar al estudiante en su proceso de aprendizaje, identificar sus dificultades y ofrecerle estrategias concretas para solventarlas. El seguimiento del alumno ha permitido comprobar la efectividad de las estrategias aplicadas y la adquisición progresiva de competencia científica.
\end{abstract}

Palabras clave: Estudio de caso | Enseñanza personalizada

Individualized Educational response to the difficulties in the process of the teaching and learning of science in Secondary Schools

Abstract: A case study whose objective is to propose new work methodologies tailored to the needs of students. Prior to their design, an in-depth literature review was conducted into the steps taken by teachers when faced with the difficulties of learning science for adolescent students. As a result, it has been considered necessary to accompany the student in their learning process, identify problems and offer specific strategies to overcome them. Tracking students has revealed the effectiveness of the strategies implemented and the progressive acquisition of scientific competencies.

Keywords: Case Study | Guided Teaching

\section{Introducción}

En el siguiente trabajo se presenta la información obtenida durante la realización de una experiencia de iniciación a la investigación en didáctica de las ciencias experimentales. En concreto, se ha profundizado en el campo de las dificultades de aprendizaje que los alumnos de secundaria pueden presentar en las áreas relacionadas con la física y la química.

La razón de esta elección tiene que ver con el pensamiento personal de que existen tantas metodologías adecuadas como alumnos, sin embargo en la práctica tan solo observamos unas pocas. Como consecuencia, lo que consideramos "tener éxito en los estudios" está reservado o limitado a aquellas personas que consiguen adaptarse a esas metodologías o a los estudiantes que responden bien ante ellas. El punto de vista de este trabajo es el opuesto. Se analiza el enfoque del conocimiento del alumno, sus inquietudes, los problemas y dificultades con los que se encuentra en el aula para alcanzar el aprendizaje de las ciencias. Por todo esto, se propone el diseño de metodologías específicas para que estos alumnos alcancen un aprendizaje significativo.

Existen alumnos con buenas capacidades para el razonamiento científico que no realizan ningún esfuerzo para intentar comprender los conceptos porque no tienen interés en la materia ya que no ven su utilidad. Otros presentan dificultades derivadas de otras áreas, como una baja competencia matemática o lingüística, que repercute en las asignaturas de ciencias. En esos casos es necesario trabajar en primer lugar sobre estos aspectos para posteriormente alcanzar el aprendizaje científico. Todo lo anterior lleva consigo que los alumnos, conscientes de que sus resultados no son buenos, se 
consideren por debajo de sus capacidades y se desmotiven todavía más con la educación en general y el aprendizaje de las ciencias en particular. De esta manera, obtienen resultados que se encuentran muy por debajo de sus capacidades.

Esto se acentúa debido a un sistema educativo organizado de manera que no se promueve una atención individualizada a los alumnos, sino que busca el punto medio del aula y a partir de ahí establece el criterio de los que van a ir bien y los que van a ir mal, perjudicando a ambos extremos. Por un lado, los que se sitúan por encima de la media limitan sus posibilidades a aprender lo que se expone en clase, lo cual resulta muy sencillo para ellos, y en consecuencia no desarrollan al máximo sus posibilidades. Si estos alumnos desarrollasen plenamente sus capacidades en las asignaturas de ciencias, aprendiendo contenidos adicionales a los del currículo oficial o realizando investigaciones propias, aprovecharían mucho mejor el tiempo, ya que no tendrían que esperar a sus compañeros. Además, podrían formar parte de metodologías más libres, en las que pudiesen decidir de alguna manera qué aprender, lo que probablemente aumentaría su motivación hacia estas asignaturas, así como un aprendizaje significativo.

Por otro lado, encontramos un grupo igual de numeroso de alumnos que se encuentra por debajo de la media. Estos deben realizar esfuerzos adicionales para cumplir objetivos impuestos mediante una metodología impuesta, les convenga o no. Los estudiantes de este grupo pasan por la escolarización recibiendo más malas noticias que buenas, mientras ven cómo algunos de sus compañeros no necesitan realizar ningún esfuerzo para superar lo que para ellos es realmente costoso y complicado. Como consecuencia su autoestima baja y en el peor de los casos se dan por vencidos, aceptando el fracaso como una consecuencia inseparable de su educación y finalmente "pasando" de todo lo que se refiere al estudio, al colegio o al aprendizaje. En este trabajo hemos decidido centrarnos en este sector más que en el anterior, aunque resultaría igualmente interesante y se presenta como propuesta de futuro.

A pesar de esta visión global tan negativa y pesimista sobre el sistema educativo, es cierto que cada vez se ponen en práctica más medidas de atención a la diversidad. Los centros utilizan distintas estrategias para cubrir estas necesidades, como los grupos de diversificación, desdobles en algunas asignaturas por nivel o capacidades, clases de refuerzo fuera del horario escolar, etc. Por un lado, estos grupos son una forma de segregación, sin embargo, gracias a ellos se genera un ritmo de aprendizaje más adaptado a cada alumno, que puede sentirse más cómodo y seguro de sí mismo. Puede que estemos en el camino correcto para conseguir motivar a nuestros estudiantes. Algunos autores por el contrario, proponen acabar con la segregación como estrategia de enriquecimiento del proceso enseñanza-aprendizaje. Lo que es seguro es que cada uno de los alumnos es diferente, aprende de una manera diferente y se inquieta por cuestiones diferentes o enfocadas desde distintas perspectivas. Los estudiantes, en este caso adolescentes, tienen que ser los protagonistas de su propio aprendizaje, en lugar de adaptarse a metodologías o estrategias educativas que se imponen en el aula como verdaderas protagonistas. Cada proceso de enseñanzaaprendizaje debería seguir su propio ritmo, con su propio estilo, de manera que potenciase las capacidades individuales de cada persona, que pudiese encontrar aquello que sabe hacer mejor y especializarse en ello para su realización personal, felicidad y como servicio al resto de la sociedad. Por supuesto, este pensamiento puede resultar utópico, sin embargo debería estar presente cada vez que nos preguntásemos hacia dónde queremos que vaya la educación de las futuras generaciones. Con esta intención se ha desarrollado el presente trabajo. 


\section{Marco teórico}

El marco teórico de este trabajo engloba: La atención a la diversidad, el alumno en la etapa de secundaria y las dificultades que encuentra en el aprendizaje de las ciencias.

\section{1- Atención a la diversidad}

Distintos autores han definido la diversidad atendiendo a varios enfoques. De acuerdo con Echeíta (1999), la polisemia del término diversidad conduce a que pueda ser abordado desde distintos puntos de vista. Sin embargo, la acepción más divulgada es la adoptada por la LOGSE, la cual se expresa mediante los términos comprensividad, en cuanto a ofrecer una única respuesta curricular a todos los alumnos, y diversidad, mediante la adecuación de este currículum común a las necesidades de cada uno (siempre que sea posible, a través de adaptaciones no significativas).

Para otros autores (Oliver Vera, 2003), el modelo educativo que atiende a la diversidad es aquel que acepta las diferencias de sus alumnos, sea cual sea su causa u origen (estilo cognitivo, capacidad para el aprendizaje, etnia, género y también las de origen social como las relativas a códigos y lenguaje, valores y modelos culturales o circunstancias socioeconómicas). Por tanto, podemos entender la atención a la diversidad como un mecanismo de ajuste de la oferta pedagógica a las capacidades, intereses y necesidades de todos los alumnos, es decir, como elemento compensador de posibles desigualdades en las condiciones de acceso a la cultura básica, o dicho de otra manera, la educación.

Además, la atención a la diversidad puede servir como eje de un conjunto de valores educativos como la aceptación y el respeto de las diferencias individuales, perfilando un concepto de diversidad basado en la equidad, en el que se sitúa el movimiento de la escuela inclusiva (Echeíta, 1999). Este podría definirse como un tipo de educación en la diversidad en el que se lleva a cabo un proceso de construcción del conocimiento surgido de la interacción entre personas distintas en cuanto a valores, ideas, percepciones, intereses, capacidades, y estilos cognitivos y de aprendizaje. Así, se favorecerían la construcción consciente y autónoma de formas personales de identidad y pensamiento. Además, se ofrecerían estrategias y procedimientos educativos de enseñanza-aprendizaje diversificados y flexibles con la doble finalidad de dar respuesta a una realidad heterogénea y de contribuir a la mejora y el enriquecimiento de las condiciones y relaciones sociales y culturales. (Jiménez y Vilá, 1999). En la práctica, implica la presencia de alumnos de todo tipo en el aula, es decir, una eliminación total de la segregación como estrategia de enriquecimiento del propio proceso de enseñanza-aprendizaje. Sin embargo, no sería viable con grupos tan numerosos como los que encontramos en la actualidad porque sería imposible proporcionar la atención individualizada que requieren algunos alumnos.

\section{2- El alumno en la etapa de secundaria. Desarrollo psicológico-emocional}

Si revisamos la bibliografía en busca de un significado acertado de la palabra adolescencia, nos encontramos con una reflexión interesante, propuesta por varios autores (Papalia y Wendkos, 1995), quienes cuestionan la autenticidad de esta etapa como un periodo de desarrollo evolutivo bien diferenciado o como una construcción artificial consecuencia de la organización cultural y social en la que vivimos. Según Palacios y cols. (2006), la adolescencia tal y como la conocemos es, hasta cierto punto, un producto del siglo XX. 
Por adolescencia nos referimos a la etapa de vida que comienza a los 12-13 años y termina alrededor de los 20 años. Se entiende como un periodo de transición en el que todavía no se es adulto, pero existe una diferencia significativa con el comportamiento y estadio propios de la infancia. Incluso en la actualidad existe controversia en cuanto al sentido y significado psicológico de la palabra adolescencia. Algunos autores la consideran como una etapa de tensión e inestabilidad, mientras que para otros representa un periodo de felicidad que no cuenta con demasiadas dificultades. En cualquier caso, la adolescencia se caracteriza por ser un período de cambios a nivel psicológico y emocional. Antes de la pubertad podemos diferenciar en niñas y niños rasgos y características que van definiendo su forma de ser y actuar. Sin embargo, es de esperar que todos los cambios físicos, cognitivos y sociales que tienen lugar en la adolescencia afecten a la personalidad y produzcan alteraciones respecto a la infancia. En esta etapa se busca una respuesta a la pregunta de ¿Quién soy yo? y se perfila la imagen que uno tiene de sí mismo mediante el desarrollo del autoconcepto, la autoestima, la búsqueda de la identidad personal y de un proyecto de vida. Como factores que influyen más significativamente en el logro de la identidad encontramos el contexto familiar, el entorno social y cultural o el género (Palacios y cols., 2006).

Por último, también se producen cambios a nivel social favoreciendo las relaciones entre iguales y ganando autonomía frente a las figuras de referencia.

Todos los factores contemplados hasta el momento constituirán los puntos de influencia del desarrollo psicológico y emocional de los alumnos que vamos a encontrar en el aula de secundaria. Cualquiera de las variables comentadas, aunque se haya afrontado superficialmente, puede afectar en gran medida al correcto desarrollo de los adolescentes y habrá que tenerlo en cuenta ya que va a repercutir en todos los ámbitos de su persona, entre ellos el que conforma nuestra responsabilidad más directa, es decir, su aprendizaje.

Si situamos el conjunto de transformaciones que se producen en la adolescencia en el contexto educativo, es al inicio de esta etapa de desarrollo donde los alumnos experimentan el cambio de la etapa educativa de primaria a secundaria. La reflexión realizada por Palacios y cols. (2006) sobre este tema es muy interesante ya que analiza las causas de la disminución de rendimiento académico que en numerosos casos acarrea esta transición educativa. Aunque tradicionalmente se suele asociar con los cambios que experimentan los propios adolescentes, Eccles ha puesto de manifiesto en sus investigaciones (Eccles y cols., 1993; Eccles y cols., 1997) la responsabilidad del sistema educativo por su incapacidad para ajustarse a las nuevas necesidades del adolescente. Los cambios que supone la educación secundaria respecto a la primaria muestran una clara asincronía con los cambios por los que está pasando el adolescente. En las clases de secundaria, en general, el profesor impone mayor control y disciplina, los contenidos están muy estructurados y ofrecen poca libertad de elección y toma de decisiones a los alumnos en relación con su propio aprendizaje. Otra modificación importante es el aumento de competitividad que clasifica a los alumnos en función de los resultados académicos. Las clases de desdoble o programas específicos para alumnos con dificultades de aprendizaje son un ejemplo de esta clasificación, que aunque tiene sus cosas buenas, puede suponer un descenso en la autoestima de los estudiantes. Todos estos factores repercuten en la motivación de los adolescentes por la educación, que desciende afectando negativamente al nivel de aprendizaje. Sin embargo, no todos los alumnos experimentan este descenso de la misma manera, algunos factores favorecen la adaptación, como una alta autoestima, un adecuado sentimiento de competencia personal, $\mathrm{o}$ un buen rendimiento académico en la etapa de primaria. También las características del centro educativo y el comportamiento y estilo docente de los profesores pueden influir. 
En esta línea, numerosos autores han descrito el papel del docente en el aula, enfocándolo desde distintos puntos de vista. Si consideramos la motivación como un ingrediente básico para el aprendizaje significativo podemos encontrar, entre otras propuestas, la teoría sobre la motivación humana de Maslow, que se explica mediante una jerarquía de necesidades organizadas en forma de pirámide (Maslow, 1991).

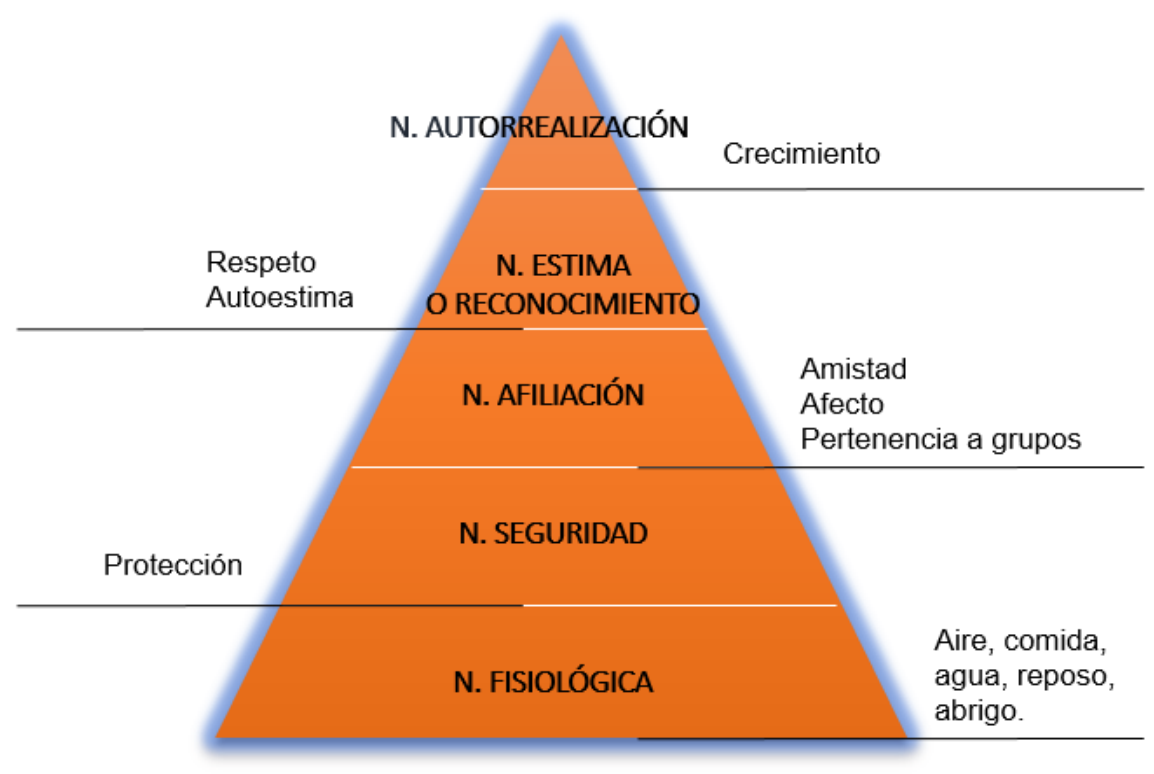

Figura 1.- Jerarquía de necesidades de Maslow

La aportación de esta teoría a la educación consiste precisamente en la jerarquización de estas necesidades ya que, según Maslow (1991), unas son predominantes sobre las otras por lo que mientras las más básicas no estén aseguradas, no van a surgir las superiores. El cambio de una necesidad a otra no es brusco, sino que va surgiendo gradualmente la siguiente conforme la anterior está siendo satisfecha.

Llevado a la práctica, es difícil que un alumno esté motivado por aprender (necesidad de autorrealización) cuando alguna de las necesidades inferiores está claramente dañada, ya sea la fisiológica, una autoestima baja, etc.

Un sistema educativo coherente con esta teoría debería tomar medidas para satisfacer las necesidades inferiores y conseguir que la motivación de los alumnos se dirigiese a su crecimiento personal y autorrealización, e incluso llegados a ese punto, según Maslow (1991), no valdría cualquier actuación docente para conseguir un aprendizaje por parte de los alumnos. Según este autor "toda la vida es educación, todo el mundo es maestro, y todo el mundo es siempre alumno" (Maslow y Lowry, 1979). Eso mismo refleja la frase: "si pienso en las grandes experiencias educativas de mi vida, las que más me enseñaron, serían las que me mostraron qué clase de persona era yo... mi matrimonio y mi paternidad. Las experiencias que me hicieron abrirme, me fortalecieron, me hicieron más alto y más fuerte, más humano" (Maslow y Hall, 1968).

Desde este punto de vista, la propuesta metodológica de Maslow y otros autores como Kohl (1969), para la educación de los jóvenes está dotada de un carácter claramente humanístico y consiste en el uso de aulas abiertas donde el profesor renuncia a su papel autoritario, habla a los alumnos como iguales y se deja llevar por sus intereses. Las investigaciones muestran que los niños aprenden mejor de los adultos que son creativos y espontáneos, que ayudan, que les aportan significados y no simplemente hechos, que poseen una alta estima de sí mismos y que ven su trabajo como liberar y 
no como controlar. El enfoque de Maslow se centra el despertar de la confianza en uno mismo, la espontaneidad y el crecimiento, además de destacar que todas las personas autorrealizadas son siempre creativas en algún ámbito, ya sea artístico, científico u otros. La creencia de Maslow sobre la educación es que debería enseñar a aprender sobre el crecimiento personal, qué elegir y qué rechazar. Leonard (1968) se une a esta visión ya que tanto él como Maslow opinan que la capacidad creativa del cerebro puede ser infinita. Leonard escribió sobre los aprendices libres y los estudiantes estimulados, los padres y educadores, defendiendo una nueva visión del aprendizaje con entornos capaces de crear el placer de aprender.

\section{3- Dificultades en el aprendizaje de las ciencias}

La adolescencia no solo conlleva grandes cambio a nivel físico y relacional sino que se extiende a nuevas formas de pensamiento que conducirán a los alumnos a concebir los fenómenos de una manera distinta a como lo hacian hasta ahora, es decir, con más autonomía y rigor en el razonamiento. A este tipo de pensamiento lo llamamos pensamiento formal y corresponde al estadio piagetiano de las operaciones formales, el cual comienza a desarrollarse alrededor de los 12 años y se consolida hacia los 1415, aunque existen investigaciones que demuestran que algunas personas no lo alcanzan nunca. Las características de este tipo de pensamiento, según Palacios y cols., (2006), son la concepción de la realidad como un subconjunto de lo posible, el desarrollo del pensamiento hipotético-deductivo, la adquisición del esquema de control de variables y el carácter proposicional del pensamiento, que se relaciona con los dos primeros ya que es la expresión de afirmaciones de naturaleza abstracta e hipotética, por ejemplo, trabajar con $\mathrm{x}$ e y en lugar de con magnitudes concretas. Además, Inhelder y Piaget (1955) establecen otra característica que es la de disponer de esquemas operacionales formales que integran el nuevo conocimiento con su experiencia.

Por otro lado, investigaciones posteriores a Piaget encuentran puntos de desacuerdo con este, en cuanto a la universalidad del pensamiento formal, la edad de adquisición de este o la poca relevancia que se le da al contenido en favor de las estructuras o esquemas mentales. El propio Piaget (1970) introdujo algunas modificaciones a su teoría, por ejemplo, sugirió que cuando el contenido no forma parte de los intereses de los alumnos, estos tienden a utilizar en mayor medida un pensamiento propio del periodo de operaciones concretas, y al contrario cuando la actividad pertenece a los intereses del estudiante o aquello para lo que tiene mayor capacidad, es decir, "cada persona alcanza el nivel de funcionamiento formal en el dominio en el que se ha especializado y mejor conoce" (Palacios y cols., 2006). La principal implicación de esta afirmación es que, contrariamente a lo que expuso Piaget inicialmente, el contenido de una tarea influye en su resolución.

En este apartado desarrollaremos brevemente algunas de las causas propias de la forma de pensar de los alumnos que pueden generarles dificultades en el aprendizaje de las asignaturas de ciencias, haciendo una breve reflexión final sobre otras causas más externas a ellos.

La primera de ellas, sobre la que se ha realizado una amplia investigación, es la que se refiere a las ideas previas, las cuales podemos entender como concepciones alternativas que los alumnos utilizan para auto-explicarse el conocimiento científico. En ocasiones estas ideas interfieren en el aprendizaje significativo, sin embargo se ha sugerido la posibilidad de verlas como ideas dinámicas formadas a partir de suposiciones implícitas que los alumnos formulan en el acto (Talanquer, 2005). Así pueden servir como base a partir de la cual adquirir el saber científico "verdadero", es 
decir, prestar atención a estas suposiciones y a partir de ellas construir el conocimiento científico. Algunas de las concepciones alternativas, se basan en la utilización de estrategias de razonamiento, adquiridas en el aula o fuera de ella, en momentos inadecuados. Por esta razón, Talanquer sugiere que la labor del docente debería ir más enfocada a enseñar a los alumnos a utilizar sus recursos cognitivos correctamente que a erradicar ideas previas. Además, podemos encontrar algunas estrategias propuestas para trabajar a partir de las estas, entre las que se incluyen la creación de modelos y su exposición en el aula o promover debates que permitan detectarlas y hacer a los alumnos reflexionar sobre ellas en lugar de erradicarlas completamente (Talanquer, 2011). Un ejemplo es la idea de que en una reacción química, como la combustión de un trozo de madera, la masa no se conserva. Perceptivamente los alumnos observan que de una gran masa de madera sólo quedan las cenizas, por lo que la cantidad de materia ha disminuido. Cuando se explica que han aparecido productos gaseosos como el dióxido de carbono y el agua a la temperatura de combustión, siguen pensando que la masa de todos los productos es menor que la del trozo de madera inicial, ya que la masa de los gases es despreciable para ellos.

De acuerdo con Campanario (2000), las pautas y estrategias de pensamiento y razonamiento, hacen referencia a las respuestas que proporcionan tanto alumnos como profesores ante una situación problemática, abordadas con escaso rigor crítico, lo que en ocasiones hace incurrir en errores. Carrascosa y cols., (1985) lo denominaron metodología de la superficialidad. En su investigación, las respuestas proporcionadas por los sujetos se caracterizaban por ser rápidas con una comprensión superficial del enunciado, incluso aunque el cuestionario que utilizaban invitaba al pensamiento razonado. Este hecho puede ser el resultado de la propia evolución, ya que nuestros antepasados debían encontrar respuestas rápidas a problemas de supervivencia que surgían instantáneamente, sin embargo, no son una estrategia adecuada en el caso del razonamiento científico. Por ejemplo, en la formulación de compuestos inorgánicos binarios, que es automática, los alumnos, pensando que un ejemplo es como otro anterior o muy parecido a todos los demás, contestan rápidamente lo primero que se les ocurre en lugar de razonarlo por un momento, y de esta manera cometen errores a pesar de que saben formular o nombrar el compuesto.

En tercer lugar, podemos observar que los alumnos tienen concepciones y creencias propias sobre la naturaleza de la ciencia y el conocimiento científico y también sobre sus procesos y productos del aprendizaje, es decir, los alumnos tienen sus propias concepciones epistemológicas. De acuerdo con Larochelle y cols., (1991), estas están presentes no solo en estudiantes de secundaria, sino también en universitarios que han cursado estudios científicos a mayor nivel, e influyen en el aprendizaje de los sujetos de manera significativa. Estas concepciones epistemológicas implican que los alumnos tienen sus propias creencias sobre el origen del conocimiento científico, generalmente asociado a situaciones concretas y sin apreciar el carácter global de la ciencia, lo que puede venir motivado por el hecho de que el saber científico se proporcione en el aula como un conjunto de verdades absolutas para explicar fenómenos individuales en lugar de hacerles ver la naturaleza dinámica y en continua revisión del conocimiento científico.

Por otro lado, la metacognición consiste, según Flavell (1976) en el conocimiento sobre los propios procesos y productos cognitivos, es decir, la consciencia propia acerca del éxito o fracaso del aprendizaje, y donde se encuentran más dificultades. Este aspecto puede interferir negativamente en el aprendizaje de los alumnos en el caso de que no cuenten con destrezas metacognitivas, es decir, cuando un alumno cree que ha aprendido y en realidad presenta evidentes dificultades en el aprendizaje, o en el otro extremo, cuando un alumno que ha aprendido correctamente sigue 
pensando que presenta dificultades. Tener destrezas o habilidades metacognitivas consistiría en tener la capacidad de reflexionar sobre el propio aprendizaje, conocer las capacidades de uno mismo y su regulación. Saber qué se sabe y no se sabe en lugar de saber o no saber. Entre las causas de dificultades, esta es más general que las anteriores y puede relacionarse de alguna manera con la autoestima, sin embargo, va un poco más allá, ya que tal y como se presenta es algo más objetiva, aunque dependa del pensamiento que tenga el alumno de sí mismo, concretamente en el ámbito escolar. Un alumno con pocas destrezas metacognitivas cuantificará su nivel de aprendizaje en función de su autoestima en este ámbito y de los resultados que el profesor le proporcione desde fuera, es decir, sus calificaciones. Sin embargo, un alumno con destrezas metacognitivas, podrá elaborar un juicio crítico para saber si ha aprendido o no independientemente de sus calificaciones y de cómo se valore a sí mismo en el ámbito académico. Por lo tanto, parece importante enseñar a los estudiantes a reflexionar sobre su aprendizaje, con el objetivo de que sean conscientes de manera realista de sus propias dificultades como primer paso para solucionarlas, y en el otro extremo, para dar confianza a los alumnos que creen que no aprenden porque tienen un concepto negativo de sí mismos, o demasiado autoexigente.

Por último, otro aspecto que genera dificultades en el aprendizaje de las ciencias es un bajo nivel en competencia matemática, que es la herramienta básica de la que se sirve el conocimiento científico. En algunos casos, la dificultad no se encuentra en las matemáticas en sí, sino en la incapacidad para transferirlas a las asignaturas de Ciencias Naturales, ya que les cuesta comprender que el simple hecho de despejar una incógnita como puede ser la masa es, en esencia, lo mismo que despejar una $\mathrm{x}$, pero dotado de significado.

Relacionando el conocimiento científico con el desarrollo personal, Pujol (2003) expresa que entender la complejidad del mundo actual y adquirir elementos para intervenir en él es incompatible con la concepción de un currículo estático; es necesario que éste se construya permanentemente sobre la base de una actitud abierta y reflexiva...". Este modo de trabajar supone: que los alumnos se impliquen en la búsqueda de respuestas a preguntas orientadas científicamente; que den prioridad a las evidencias; que formulen explicaciones; que conecten las explicaciones con el conocimiento científico y que comuniquen y justifiquen dichas explicaciones. En este proceso el maestro es quien debe diseñar las estrategias para que el alumnado desarrolle estas habilidades y progresivamente se vaya haciendo cada vez más autónomo (Eduteca, 2005; Pujol, 2003). En cualquier caso, debe quedar claro que las causas del fracaso no deben situarse únicamente en los alumnos.

Como conclusión, podemos destacar que existen infinidad de factores que influyen en cómo aprenden nuestros alumnos. A pesar de que es imposible controlarlos todos un objetivo alcanzable sería estudiar el caso individualizado de cada alumno, empezando por conocerle y comprender su comportamiento y motivaciones desde el contexto en el que se sitúa, como primer paso para inferir su modo de aprendizaje y comenzar a trabajar con él o ella.

\section{Objetivos}

Los objetivos que se pretenden conseguir en este trabajo son los siguientes:

- Proponer metodologías específicas que se adapten al modo de aprendizaje de un alumno concreto, basándonos en la afirmación de que cada alumno es diferente, y por lo tanto aprende de diferente manera. 
- Promover el aprendizaje significativo mediante la aplicación de algunas de las metodologías propuestas para ayudar a la alumna a superar las asignaturas de ciencias con éxito.

- Realizar un seguimiento en el tiempo de las propuestas metodológicas aplicadas para analizar su funcionalidad.

- Extraer conclusiones de los resultados obtenidos que permitan generalizar el caso estudiado, así como proponer nuevas líneas de investigación para el futuro.

\section{Método}

Se ha realizado un estudio de casos en el que se han analizado a fondo las causas de las dificultades en el aprendizaje de las asignaturas de ciencias para tres alumnas. A continuación, se han propuesto diferentes metodologías para cada una de ellas con el objetivo de hacer hincapié en el trabajo de aquellos aspectos en los que se detecta mayor dificultad. La finalidad es trasladar al aula la idea de que debe ser la enseñanza la que se adapte a los alumnos y no al contrario. En la medida de lo posible, la metodología propuesta se ha llevado a la práctica y se han analizado sus resultados. En el presente artículo se muestra uno de los tres casos.

\section{1- Selección de la participante del estudio}

Para que las propuestas realizadas puedan ser aplicadas con relativa frecuencia en el aula, se ha decidido seleccionar casos generales, es decir, aquellos cuyas causas de dificultad en el aprendizaje se observan con frecuencia en la etapa de secundaria y no están demasiado sujetas a circunstancias personales del entorno de los alumnos, aunque esto es estrictamente imposible ya que no se pueden separar y aislar los factores que influyen en el comportamiento de un ser humano en general, y el rendimiento de un alumno en particular. Para la selección de los participantes los profesores de ciencias del centro propusieron varios casos. La elección final se realizó teniendo en cuenta la opinión de los tutores.

\section{2- Las entrevistas}

Realizada la selección se han mantenido entrevistas con tutores, profesores de ciencias y las propias alumnas, con el objetivo de recopilar la máxima cantidad de información posible enfocada desde distintos puntos de vista. Se pretende conocer los datos que puedan ser de ayuda para saber por qué estas alumnas rinden por debajo de sus capacidades, centrando la atención en el proceso de enseñanza-aprendizaje, en lugar de su situación vital, que será importante no obstante para contextualizar cada uno de los casos. Es importante señalar que con este análisis no se ha pretendido diseñar y aplicar una terapia en la que se solventen problemas personales de las alumnas, incluidos los académicos, sino proporcionar una ayuda y apoyo en el estudio para que sean capaces de realizar un aprendizaje significativo en las asignaturas de ciencias que les permita superarlas con éxito.

Por un lado, mediante la entrevista mantenida con los tutores se busca una opinión global y generalizada, ya que estos conocen la trayectoria del alumno en todas las asignaturas y sus capacidades de forma general, así como su estado vital de desarrollo, su personalidad, relaciones sociales, etc.

Por otro lado, la razón de realizar una entrevista con los profesores de ciencias es obtener la misma información con una perspectiva diferente, es decir, en el caso 
concreto de las asignaturas de ciencias, e informarles de aspectos prácticos del trabajo, así como solicitar su ayuda y permiso para la posterior aplicación.

Al inicio de la primera entrevista mantenida con las alumnas se busca informarles del estudio que se va a realizar, desde un punto de vista tal que las convierta en cómplices más que en objetos de estudio. Para ello, a lo largo de la conversación se solicita su ayuda para formar una opinión que englobe todos los puntos de vista sobre el estado de la educación en la actualidad y propuestas de mejora para hacer las asignaturas de ciencias más motivantes, entretenidas o fáciles de entender. De esta manera, es más sencillo abordar el tema de las dificultades de aprendizaje con las que ellas se encuentran, en lugar de analizar directamente el porqué de su bajo rendimiento, donde pueden sentirse más atacadas y expresarse con menos libertad. En la primera parte de la entrevista se habla de sus aficiones extraescolares y a que les gustaría dedicarse en el futuro, para eliminar la tensión inicial y conocer su contexto extraescolar en lo que se refiere a sus inquietudes. En la segunda parte se aborda el tema de la educación actual, analizando los puntos débiles que encuentran y cómo mejorarlos desde un punto de vista práctico y realista llegando al final de la entrevista a las carencias que encuentran en las asignaturas de ciencias, y por qué en ocasiones les resulta complicado comprender su contenido, comentando todos los aspectos que influyen; desde el papel del docente, el ambiente del aula y las características del contenido hasta su propia actitud hacia el aprendizaje de la materia. Por último, se ha pedido que expresen cómo creen que se les podía ayudar a demostrar todo su potencial en las asignaturas de ciencias y qué estrategias piensan que les podrían venir bien para conseguir este objetivo.

\section{3- Diseño de metodologías específicas}

Con la información recogida a partir de las entrevistas y de la observación de las alumnas en el aula, se han sugerido estrategias metodológicas para cada uno de los tres casos trabajados, que se llevarán a la práctica dentro o fuera del aula en función de los requerimientos de la propuesta. En el presente artículo, se presentan el análisis y resultados de uno de los casos.

\section{Caso de estudio}

El aula de tercero de ESO en la que encontramos a esta alumna está formada por 22 estudiantes con niveles cognitivos muy heterogéneos.

Se trata de una clase con facilidad para distraerse donde los alumnos muestran un interés bajo. La distribución de mesas es acorde con esto ya que se sitúan individualmente y muy separados unos de otros. Por eso, la metodología de los profesores con el grupo se basa en minimizar la participación de los alumnos. De esta manera, se ha conseguido que su comportamiento sea adecuado pero es muy difícil saber si un alumno está comprendiendo el contenido o si se encuentra motivado para el aprendizaje, ya que la interacción es escasa. Académicamente, encontramos tres grupos de estudiantes bien diferenciados. El primero obtiene buenos resultados sin ningún problema. El segundo está formado por seis alumnos que salen de clase en los desdobles bajos. El tercer grupo, consiste en unos pocos alumnos que sin encontrarse en el grupo de desdoble, están obteniendo malos resultados académicos. Nuestro caso se centra en una alumna de este grupo.

La estudiante tiene actualmente suspendida la asignatura de Física y Química. Su comportamiento en clase es, como el de todos los demás, bueno a la fuerza, por la actitud que adoptan los profesores en el aula. Es difícil conocer su motivación o interés 
por el aprendizaje únicamente mediante la observación, ya que prácticamente no existe participación espontánea. Su propia opinión acerca de la educación es indiferente. Su relación con los compañeros es buena, se lleva bien con casi todos y tiene varias amistades. Por otro lado, según su tutora y su profesora de ciencias, tiene una baja autoestima, lo que se demuestra en el hecho de que antes de leer un problema ya está segura de que no va a saber resolverlo, o que necesita continuamente la aprobación del profesor cuando hace o corrige un ejercicio para poder continuar. Respecto al trabajo autónomo, a pesar de que cuenta con un profesor particular, la mayoría de los días no realiza la tarea de casa, sino que la hace deprisa y corriendo al inicio de la clase.

Encuentra la educación demasiado estática, y aunque reconoce que en su clase sería muy complicado implantar otra metodología de trabajo, preferiría métodos más dinámicos para favorecer el aprendizaje, en concreto, más juegos, más trabajo manual y más trabajos de investigación. Por otro lado, cree que hay poca optatividad en la etapa de secundaria y que le gustaría poder elegir antes qué asignaturas cursar y cuáles no. Cuando acabe secundaria, quiere emprender estudios de artes, y señala que no existe ninguna asignatura en los tres primeros cursos de la etapa que incluya contenidos de historia del arte, por ejemplo, que le resultan muy interesantes.

Respecto a las asignaturas de ciencias, es decir, Física y Química, Biología y Geología y Matemáticas, encuentra problemas especialmente en la primera y la última ya que sus contenidos requieren mayor esfuerzo cognitivo. Tanto ella como su profesora de ciencias coinciden en que su dificultad principal es comprender y realizar los problemas, sin embargo, cada vez que consigue entender algún concepto de cierta complejidad su motivación e interés aumenta, y encuentra entretenido realizar más ejercicios sobre ello, porque sabe que le van a salir bien. Después de este pico de motivación vuelve a decaer poco a poco hasta su estado inicial. Un aspecto positivo es que tal y como ella misma expresa, durante este curso ha aprendido a estudiar con lápiz y papel. Antes leía los problemas y entendía los pasos que se llevaban a cabo para su resolución, sin embargo, al llegar al examen no era capaz de reproducirlo ya que no los había practicado. Actualmente comenta que para estudiar realiza los ejercicios por sí misma en lugar de limitarse a observarlos.

\section{1- Detección de las causas de sus dificultades}

En primer lugar se observa poco interés y escasa motivación por parte de la alumna para el aprendizaje de las ciencias. La dificultad que entraña y el esfuerzo de abstracción que requiere un contenido que, a ojos de la estudiante, no tiene ninguna aplicación, provoca un desinterés bastante acentuado que ella misma expresa y que se deja ver mediante la actitud pasiva que adopta en el aula en dichas asignaturas. De esta manera, podríamos situar la primera causa de las dificultades en la propia desmotivación, que hemos expuesto en la fundamentación teórica y que actúa como una barrera que se cierra ante la llegada del contenido científico, especialmente cuando requiere un esfuerzo cognitivo para su comprensión.

Mediante la observación en el aula se han detectado otras causas que pueden contribuir a los malos resultados. La mayoría de los alumnos del grupo realizan una escucha activa en clase destacando en el texto las palabras clave, o realizando anotaciones. Por el contrario, su actitud pasiva favorece una adquisición superficial del contenido que olvidará con facilidad. Por lo tanto, podríamos expresar la segunda causa de dificultad en la ausencia de escucha activa. Además, sus estrategias de estudio de contenido teórico o memorístico siguen siendo pasivas, sin realizar esquemas o resúmenes para estructurarlo. 
Por último se detecta una dificultad especial en la resolución de problemas, que ha sido expresada por la profesora de ciencias, por la alumna, y también observada en clase. Mediante el acompañamiento en el aula se ha atribuido esta dificultad a las pautas de pensamiento de la estudiante al enfrentarse a los ejercicios. Es probable que la falta de motivación provoque un pensamiento superficial y el uso de "atajos cognitivos" para resolver los problemas con el fin, más o menos consciente, de terminarlos cuanto antes y pasar a otra cosa. Por otro lado, la baja autoestima de la alumna incorpora estos resultados como una prueba explícita que justifica su inseguridad y contribuye a que no desaparezca.

\section{2- Propuestas metodológicas}

Entre las propuestas de la alumna en la primera entrevista, destacó que le parecía importante que los profesores adopten la actitud de hacer pensar a los alumnos acerca de las dudas que tienen. Es decir, en lugar de simplemente responder ante la pregunta de un alumno, actuar como guía mediante otras preguntas o a través de pistas para que él mismo sea capaz de responderse sobre su duda inicial. Para esto, es importante dar tiempo a los alumnos tanto en el momento en el que están pensando como cuando se están expresando.

Por nuestra parte se proponen otras medidas en base a las dificultades detectadas, dejando parcialmente al margen las que no son provocadas por las asignaturas de ciencias, es decir la baja autoestima y la desmotivación por el aprendizaje, aunque se realiza un refuerzo positivo de los logros de la alumna y se minimiza la importancia de sus errores.

En primer lugar, es importante que la alumna adquiera técnicas de estudio y se le proporcionen las herramientas necesarias para promover la escucha activa, como por ejemplo el subrayado, tomar apuntes, etc. con el objetivo de favorecer la adquisición del contenido en el aula y la detección por su parte de aquello que le cuesta más comprender, de manera que pueda preguntarlo en ese mismo momento y escucharlo una vez más. Esta alumna responde a la presión que causa la calificación, probablemente debido a la ausencia de motivación intrínseca, por lo que darle una contribución en la nota final a aspectos como por ejemplo presentar esquemas de las unidades didácticas junto con la entrega del cuaderno de trabajo, podría ser un primer paso para conseguir que los llevase a cabo. Es evidente que aún mejor que lo anterior, es conseguir aumentar la motivación intrínseca de la alumna. Sin embargo, ella misma en la entrevista, propone algunas claves de las que se ha extraído la información pertinente para realizar nuevas propuestas. Existen muchos factores que influyen en el gusto de una asignatura, por ejemplo el profesor o el contenido. En el segundo caso, suele gustar más a los estudiantes cuando es cotidiano y reconocible para ellos. Estamos rodeados de física y química miremos donde miremos. Sin embargo, si preguntas a los alumnos, ninguno te responderá que química es el móvil con el que se comunica con sus amigos, o la ropa que lleva. Probablemente responderán que los estados sólido, líquido y gaseoso del agua son los fenómenos científicos más cercanos que reconocen. Plantear una metodología aplicada, en la que se reconozca la ciencia en el día a día puede ser una de las claves para conseguir alumnos motivados para su aprendizaje.

Por último, para resolver su problema con los ejercicios numéricos se propone fragmentarlo en ejercicios más pequeños, que lleven a la alumna metódicamente hasta el resultado correcto lo que se puede aportar, por ejemplo, en forma de pautas o de clave dicotómica. En ambos casos, aunque especialmente en la lista de pautas, hay que tener cuidado para evitar un problema mayor que es que la alumna resuelva 
los ejercicios como quien prepara una receta sin cuestionar su sentido o significado, por lo que estas pautas se deben proporcionar de manera abierta para favorecer el razonamiento de la estudiante. Por ejemplo, en el caso de la formulación de compuestos inorgánicos binarios se propone la siguiente secuencia:

- Escribe los símbolos de los elementos.

- Piensa en qué orden deben colocarse, sabiendo que el de menor carácter metálico se escribe a la derecha.

- Escribe en la parte superior de cada elemento el número de oxidación con el que actúa.

- Intercambia los números de oxidación entre los dos elementos, colocándolos como subíndice.

- Si puedes, simplifica.

\section{4- Aplicación}

En este caso, se han aplicado algunas propuestas dentro del aula de manera individualizada, y se ha contado con una persona de apoyo en varias sesiones de las asignaturas de Física y Química y Matemáticas.

La primera estrategia ha consistido en proporcionar herramientas para realizar una escucha activa y el análisis conjunto de algunos párrafos del libro de texto con el fin de seleccionar la información clave que contienen. Esto se ha llevado a cabo de manera paralela al transcurso de las explicaciones del profesor, intentando seguir el mismo ritmo, aunque en ocasiones ha sido necesario detenerse en algún punto en el que la alumna encontraba problemas de compresión.

Por otro lado, se ha insistido en la resolución de ejercicios, en el caso de Física y Química para la formulación de compuestos inorgánicos binarios, y en el caso de Matemáticas con distintos conceptos relacionados con las funciones. Para ambas materias, se han proporcionado pautas correspondientes a los ejercicios-tipo realizados. En primer lugar se propone la lectura del enunciado y se subrayan por un lado las palabras clave y los datos importantes, y por otro lado el objetivo, es decir, qué dato se debe dar como resultado. A continuación, se lleva a cabo la resolución del ejercicio utilizando las pautas proporcionadas siendo necesario escribir cada uno de los pasos sin saltarse ninguno. Para comprobar que la alumna no se ha limitado a aplicar la receta, una vez los errores han disminuido, se combinan estos ejercicios con otros de carácter más abierto, lo cual resultó sencillo en Matemáticas, pero no tanto en ejercicios de formulación ya que el problema acaba cuando formulas o nombras el compuesto. Sin embargo, se puede cuestionar a qué familia de compuestos pertenece (óxido, sal binaria, hidruro, etc.), el tipo de enlace o de compuesto de que se trata (molécula discreta, red cristalina, etc.).

Todo lo anterior ha ido acompañado de refuerzo positivo por la complicación que en este caso presenta la baja autoestima de la alumna. Por otro lado, se ha gestionado el error de forma que la alumna lo analiza y utiliza para construir la respuesta correcta a partir de este, evaluando en qué momento se había cometido y por qué.

\section{Resultados}

Los resultados se expresan en base a lo observado en el aula durante el transcurso de las sesiones en las que se ha contado con una persona de apoyo para la comprensión del contenido y la resolución de problemas. 
La metodología aplicada ha tenido como objetivo principal que la alumna encontrase menos dificultad en la resolución de problemas. Las pautas proporcionadas para ello han aumentado la seguridad de la alumna en sí misma para la resolución de ejercicios. Inicialmente se observaba la necesidad de reconocimiento de la persona de apoyo en cada paso aplicado para llegar a la solución sin errores. Gradualmente esta necesidad ha disminuido al aumentar la seguridad de la alumna que ha sido capaz de resolver los ejercicios por sí misma sin demasiados errores utilizando las pautas proporcionadas. El siguiente paso sería ser capaz de realizar cualquier ejercicio sin ninguno de los dos apoyos, lo que significaría que las pautas de pensamiento anteriores han sido eliminadas y se han adquirido correctamente las nuevas. En este sentido podemos concluir que la alumna ha alcanzado el objetivo principal. Sin embargo, todos los resultados han sido tomados con la presencia de una persona en el aula supervisando sus progresos, lo que evidentemente puede influir en el comportamiento, de manera que no se puede asegurar que al desaparecer esta figura los resultados continúen en esta trayectoria, ni cuánto tiempo se mantenga esta actitud. Aun así, se ha conseguido diseñar una metodología de trabajo personalizada que lleva a un resultado satisfactorio.

Generalizando el caso, se ha observado que la metodología propuesta, consistente en elaborar unas pautas que fragmenten el contenido de un problema en otros más pequeños puede ayudar a reelaborar las pautas de pensamiento de los alumnos. Por otro lado hay que tener cuidado de que esto constituya la excusa perfecta para dejar de pensar, por lo que se propone reforzar estos ejercicios de carácter metódico con otros de carácter más abierto, que no se puedan resolver mediante las pautas sino utilizando los conocimientos adquiridos.

\section{Discusión}

A la vista de los resultados encontrados en este trabajo, se subraya la necesidad de atender a la diversidad, conociendo a nuestros alumnos y siendo capaces de ofrecerles lo que necesita cada uno de ellos para potenciar al máximo sus capacidades.

Como esto es complicado en aulas con grupos numerosos, se debería potenciar la metacognición de manera que los alumnos individualmente puedan valorar y reflexionar sobre sus capacidades para el aprendizaje.

Por último se proponen, a raíz de este trabajo, distintas líneas de investigación en metodologías que atiendan a necesidades individuales de los alumnos.

En primer lugar, se sugiere la posibilidad de prolongar la parte práctica del estudio del caso durante al menos un curso para recopilar más información sobre la evolución del aprendizaje.

En segundo lugar, se plantea la elaboración de protocolos en los que aparezcan las medidas que favorecen a cada tipo general de alumnos, sean como el caso analizado en este trabajo $u$ otros adicionales.

Finalmente, se propone llevar a cabo este mismo trabajo atendiendo a aquellos alumnos incluidos en la justificación del proyecto, cuyas capacidades o cuyos resultados se encuentran por encima de la media. 
Campanario, JM. y Otero, JC. (2000). Más allá de las ideas previas como dificultades de aprendizaje: Las pautas de pensamiento, las concepciones epistemológicas y las estrategias metacognitivas de los alumnos de ciencias. Enseñanza de las ciencias, 18(2), 155-169.

Carrascosa, J. y Gil, D. (1985). La metodología de la superficialidad y el aprendizaje de las ciencias. Enseñanza de las ciencias, 3, 113-120.

Eccles, JS., Lord, SE., Roeser, RW., Barber, BL., \& Hernández-Jocefowicz, DM. (1997). The association of school transitions in early adolescence with developmental trajectories through High School. Health risk and developemental transitions during adolescence. Cambridge: Cambridge University Press.

Eccles, JS., Midgley, C., Wigfield, A., Buchanan, CM., Reuman, D., Flanagan, C., \& Maclver, D. (1993). Development during adolescence: the impact of stage-environment fit on young adolescents' experiences in schools and in families. American Psycholgist, 48, 90-101.

Echeíta, G. (1999). Reflexiones sobre Atención a la Diversidad. Acción Educativa, 102-103, 30-43.

Eduteca (2005). La Indagación en los Estándares de Ciencias. La Indagación y los Estándares Nacionales de Educación en Ciencias: Una Guía para la Enseñanza y el Aprendizaje. Washington, D.C. National Academy Press (2000). http://www.eduteka.org/lnquiry2.php/

Flavell, J.H. (1976). Metacognitive aspects of problem solving. The nature of intelligence. Hillsdale, Nueva Jersey: Lawrence Erlbaum.

Harter, S., Marold, DB., Whitesell, NR., \& Cobbs, G. (1996). A model of the effects of perceived parent and peert support on adolescent false self behavior. Child development, 67, 360-374.

Hall, MH. (1968) A conversation with Abraham H. Maslow. Psychology Today, 35-37, 54-57.

Inhelder, B., \& Piaget, J. (1955). De la logique de l'enfant à la logique de l'adolescent. París: Presses Universitaires de France.

Jiménez, P. y Vilá, M. (1999). De educación especial a educación en la diversidad. Málaga: Aljibe.

Kohl, H. (1969). The open classroom. New York: New York Review Books.

Larochelle, M., \& Désautels, J. (1991). «Of course, it's obvious»: adolescents' ideas of scientific knowledge. International Journal of Science Education, 13, 373-389.

Leonard, G. (1968). Education and ecstasy. New York: Delta.

Lowry, RJ. (1979). The journals of A. H. Maslow. Monterey, CA: Brooks/Cole.

Marcia, J. (1966). Development and validation of ego-identity statuses. Journal of Personality and Social Psichology, 3, 551-558.

Maslow, AH. (1991). Motivación y personalidad. Madrid: Díaz de Santos S.A.

Oliver Vera, MC. (2003). Estrategias didácticas y organizativas ante la diversidad. Dilemas del profesorado. Barcelona: Octaedro.

Palacios, J., Marchesi, Á. y Coll, C. (2006) Desarrollo psicológico y educación. Vol.1: Psicología evolutiva. Madrid: Alianza Editorial.

Papalia, DE y Wendkos, S. (1995). Psicología. México: Mc Graw-Hill.

Piaget, J. (1970). L'evolution intellectuelle entre l'adolecsence et l'âge adulte. Rapport sur le III Congrés International FONEME sur la Formation humaine de l'adolescent à la madurité, Milán.

Pujol, RM. (2003). Didáctica de las ciencias en la educación primaria. Madrid: Síntesis Educación.

Sanz MT., Menéndez, FJ., Rivero, MP. y Conde, M. (2013). Psicología de la motivación. Sanz y Torres. UNED.

Talanquer, V. (2005). El químico intuitivo. Educación Química, 16(4), 114-122.

Talanquer, V. (2011). El papel de las ideas previas en el aprendizaje de la química. Alambique. Didáctica de las ciencias experimentales, 69, 3541. 